\title{
Two-dimensional rogue waves on zero background in a Benney-Roskes model
}

\author{
Lijuan Guo, ${ }^{1,2}$ Jingsong He $\odot,{ }^{3,{ }^{*}}$ Lihong Wang, ${ }^{4}$ Yi Cheng, ${ }^{2}$ D. J. Frantzeskakis,${ }^{5}$ T. S. van den Bremer, ${ }^{6}$ and P. G. Kevrekidis ${ }^{7}$ \\ ${ }^{1}$ College of Science, Nanjing Forestry University, Nanjing, Jiangsu 210037, People's Republic of China \\ ${ }^{2}$ School of Mathematical Sciences, University of Science and Technology of China, Hefei, Anhui 230026, People's Republic of China \\ ${ }^{3}$ Institute for Advanced Study, Shenzhen University, Shenzhen, Guangdong 518060, People's Republic of China \\ ${ }^{4}$ School of Mathematics and Statistics, Ningbo University, Ningbo, Zhejiang 315211, People's Republic of China \\ ${ }^{5}$ Department of Physics, University of Athens, Panepistimiopolis, Zografos, Athens 15784, Greece \\ ${ }^{6}$ Department of Engineering Science, University of Oxford, Parks Road, Oxford OX1 3PJ, United Kingdom \\ ${ }^{7}$ Department of Mathematics and Statistics, University of Massachusetts, Amherst, Massachusetts 01003-4515, USA
}

(Received 20 March 2019; accepted 10 August 2020; published 8 September 2020)

\begin{abstract}
A prototypical example of a rogue wave (RW) structure in a two-dimensional (2D) nonlocal, nonlinear Schrödinger model, namely, a variant of the Benney-Roskes (BR) system, is presented. The analytical methodology involves a Taylor series expansion of an eigenfunction of the model's Lax pair, which is used to form a hierarchy of infinitely many eigenfunctions. These are used for the construction of 2D RWs of the BR system by the evenfold Darboux transformation. The obtained 2D RWs, which are localized in both space and time, can be viewed as a $2 \mathrm{D}$ analog of the Peregrine soliton.
\end{abstract}

DOI: 10.1103/PhysRevResearch.2.033376

\section{INTRODUCTION}

A two-dimensional (2D) rogue wave (RW) is a short-lived large-amplitude wave, which is doubly localized in two spatial variables $x$ and $y$ as well as in time $t$, and its modulus is a rational function. Naturally, a RW solution of a suitable 2D partial differential equation could provide a dynamical paradigm in a number of areas of physics, including nonlinear optics, atomic Bose-Einstein condensates, and ocean waves (see, e.g., the reviews in [1,2] as well as [3-6]. However, here we report an analytical form of such a genuine $2 \mathrm{D}$ RW.

A candidate physically relevant model that may give rise to purely 2D RWs is a nonlocal nonlinear Schrödinger (NLS) model, where the field obeying a NLS-type equation is coupled to a mean term. Such nonlocal NLS models may result from multiscale expansion methods in physical systems where the underlying governing equations feature quadratic nonlinearities; pertinent examples include water waves [7-10] and optical media with nonresonant quadratic nonlinearities (described by the coefficient $\chi^{(2)}$ of the medium's susceptibility tensor) [11,12]. This motivates us to study such a nonlocal NLS model, which is of the following dimensionless form:

$$
\begin{aligned}
i u_{t}+u_{x x}-u_{y y}+\left(2 \kappa|u|^{2}+S\right) u & =0, \\
S_{x x}+S_{y y}=-4 \kappa\left(|u|^{2}\right)_{x x}, \quad \kappa & = \pm 1 .
\end{aligned}
$$

*Corresponding author: hejingsong@szu.edu.cn; jshe@ustc.edu.cn

Published by the American Physical Society under the terms of the Creative Commons Attribution 4.0 International license. Further distribution of this work must maintain attribution to the author(s) and the published article's title, journal citation, and DOI.
Here $u(x, y, t)$ represents the envelope of the wave packet, so the actual wave field is given by the product of $u(x, y, t)$ and a carrier wave in the $x$ direction, while $S(x, y, t)$ describes the so-called wave-induced mean field, which in turn interacts with the surface wave (in the context of water waves) or the electric field envelope of the first harmonic (in $\chi^{(2)}$ nonlinear optical media). Furthermore, the parameter $\kappa$ sets, in general, the type of nonlinearity: $\kappa= \pm 1$ corresponds to defocusing and focusing cases. Here we will consider the case $\kappa=-1$.

We should mention that, in the absence of the mean field $S(x, y, t), \mathrm{Eq}$. (1) reduces to the $(2+1)$-dimensional NLS equation. On the other hand, in the presence of $S(x, y, t)$, Eq. (1) is a special case of a generalized Benney-Roskes system (BRS) (first reported in Ref. [7]) [see Eq. (6.51) in Ref. [13]]. In this model, the signs of the dispersion terms may also change, giving rise, e.g., to an elliptic-hyperbolic [rather than a hyperbolic-elliptic in Eq. (1)] structure. Equation (1) corresponds to a suitable choice of the various signs (and rescaling) of the BRS. For other choices of the signs, the BRS can describe water waves and can be reduced to the DaveyStewartson (DS) equation [8]. Nevertheless, for our choice of parameters, Eq. (1) does not describe water waves, as in Refs. [8-10]. Following [13], we will refer to the model at hand, Eq. (1), as a Benney-Roskes system, which is a focusing version of the original DS equation.

Evidently, the BRS under consideration is a $2 \mathrm{D}$ extension of the famous NLS equation [14,15]. The latter is known to possess $1 \mathrm{D}$ rational RWs, i.e., the fundamental RW in the form of the so-called Peregrine soliton [16-18], as well as other higher-order RWs [19-22], which have been observed in optical systems and water tank experiments [23-29]. Since the BRS can be viewed as a nonlocal hyperbolic NLS equation, it should be mentioned that its local counterpart, i.e., the 2D hyperbolic NLS, has been computationally explored in Ref. [30] 
in the context of deep water gravity waves, with the numerical results suggesting that RWs may persist in such settings.

The main focus of the present work is to offer explicit analytical solutions for BRS RWs, which can be viewed as the prototypical generalization of Peregrine solitons to genuinely higher-dimensional settings. The relevance of such settings in physical applications highlights the importance of a systematic toolbox enabling the identification of such solutions. While the importance of this problem has been recognized and other mathematically motivated (from integrability theory) 2D extensions of RWs have been proposed [31], the present 2D RW proposal constitutes a prototypical physically relevant example in view of the above remarks.

Before proceeding, we review some key properties of the BRS (1). First we note that it is a completely integrable system via the inverse scattering transform and particularly by means of the so-called $\bar{\partial}$ method [32-34]. However, although the relevant BRS has been originally developed in and considered since the late 1960s, the construction of 2D RWs for this system is still an open problem, strongly motivated by (a) identifying a 2D RW that could be suitable for physical applications and (b) finding a 2D analog of the Peregrine soliton solution.

In this work we present genuine 2D RWs of the BRS equation, decaying as $O\left(\frac{1}{r^{\prime 2}}\right)$, for a given time $t$, where $r^{\prime}$ denotes the radial distance from the center of the RW in the moving reference frame (discussed below). Unlike higher-order multipole lumps [35] whose maximum amplitude approaches a nonzero constant as $t \rightarrow \pm \infty$, here we present two kinds of solutions featuring different asymptotic behaviors for large $|t|$.

(i) The maximum value of the first-order RW decays $\sim \frac{1}{r^{\prime}}$ to 0 , which guarantees that this is indeed a RW wave "appearing from nowhere and disappearing without a trace" [18].

(ii) The RW-lump solution's maxima consist of a central peak given by a lump and an outer ring given by a RW. Once the RW disappears at large times, this solution reduces to a lump of constant amplitude (at large times).

\section{ANALYSIS}

In what follows we provide an outline of the methods and give the main results. We start by recalling the Lax pair and Darboux transformation (DT) for the BRS [36-38]. The relevant Lax pair is $[36,38]$

$$
\Psi_{y}=J \Psi_{x}+U \Psi, \quad \Psi_{t}=2 J \Psi_{x x}+2 U \Psi_{x}+V \Psi,
$$

with a constant diagonal matrix $J=\left(\begin{array}{cc}i & 0 \\ 0 & -i\end{array}\right)$ and two potential matrices

$$
U=\left(\begin{array}{ll}
0 & u \\
v & 0
\end{array}\right), \quad V=\left(\begin{array}{cc}
(w+i Q) / 2 & u_{x}-i u_{y} \\
v_{x}+i v_{y} & (w-i Q) / 2
\end{array}\right) .
$$

Here the eigenfunction $\Psi=(\psi, \phi)^{\top}$ ( $\mathrm{T}$ denotes transpose), the potentials $u, v=\kappa u^{*}(\kappa= \pm 1) \in \mathbb{C}$, the field $Q=$ $2 \kappa|u|^{2}+S \in \mathbb{R}$, and $w \in \mathbb{R}$ satisfying $w_{y}=-Q_{x}-2(u v)_{x}$ and $w_{x}=Q_{y}-2(u v)_{y}$ are functions of the three variables $x$, $y$, and $t$. As is typically the case in such integrable models, the BRS is obtained from the compatibility of the Lax pair, i.e., $\Psi_{y t}=\Psi_{t y}$. Since $S$ and $Q$ are two auxiliary functions in the BRS and its Lax pair, below we will focus on the $u$ waveform.
The construction of the $N$-fold DT of the BRS necessitates $N$ eigenfunctions $\Psi_{k}=\left(\psi_{k}, \phi_{k}\right)^{\top}(k=1,2, \ldots, N)$ and $\tilde{\Psi}_{k}=\left(\phi_{k}^{*}, \kappa \psi_{k}^{*}\right)^{\top}$, which are associated with a given "seed" solution $u$ and $v$, of the Lax pair equation (2). The $N$ th-order solution [38] of the BRS generated by the $N$-fold DT reads

$$
u^{[N]}=u+2 i \frac{\delta_{2}}{\delta_{1}},
$$

where $\delta_{1}$ and $\delta_{2}$ are two determinants of $\Psi_{k}=\left(\psi_{k}, \phi_{k}\right)^{\top}$ and $\tilde{\Psi}_{k}=\left(\phi_{k}^{*}, \kappa \psi_{k}^{*}\right)^{\top}$ (see the Appendix). The line RWs on nonzero background were constructed in Refs. [38,39]. As mentioned above, the defocusing BRS, for $\kappa=1$, has no smooth rational solutions; thus, hereafter, we focus on the focusing case and set $\kappa=-1$.

We are now in a position to construct RW solutions $u$ of the BRS starting from a zero seed solution $u=0, v=0$, $Q=0$, and $w=0$ of Eq. (4) by the DT method. It is crucial to find proper eigenfunctions $\Psi_{k}$ associated with the zero seed solution in the Lax pair equation (2) in order to find RWs. Substituting this seed back into Eq. (2), we get a basic eigenfunction $\Psi$, namely,

$$
\begin{aligned}
& \psi=\psi\left(b_{1}, x, y, t\right)=a_{1} \exp \left[i b_{1}\left(x+i y-2 b_{1} t\right)\right], \\
& \phi=\phi\left(b_{2}, x, y, t\right)=a_{2} \exp \left[i b_{2}\left(x-i y+2 b_{2} t\right)\right],
\end{aligned}
$$

where

$$
\begin{aligned}
& a_{1}=\exp \left[b_{1}\left(s_{0}+s_{1} \epsilon+s_{2} \epsilon^{2}+\cdots+s_{N} \epsilon^{N}\right)\right], \\
& a_{2}=\exp \left[b_{2}\left(s_{0}+s_{1} \epsilon+s_{2} \epsilon^{2}+\cdots+s_{N} \epsilon^{N}\right)\right]
\end{aligned}
$$

are two overall factors added intentionally in order to introduce more expansion coefficients $s_{i}$. Importantly, the two components $\psi$ and $\phi$ in the basic eigenfunction $\Psi$ are independent of each other under the condition of a zero seed solution. Performing a Taylor series expansion for the above basic eigenfunction $\Psi$ at $\left(\lambda_{1}, \lambda_{2}\right)^{\top}$, we obtain

$$
\begin{aligned}
& \psi\left(\lambda_{1}+\epsilon\right)=\psi^{[0]}+\psi^{[1]} \epsilon+\cdots+\psi^{[N]} \epsilon^{N}+O\left(\epsilon^{N+1}\right), \\
& \phi\left(\lambda_{2}+\epsilon\right)=\phi^{[0]}+\phi^{[1]} \epsilon+\cdots+\phi^{[N]} \epsilon^{N}+O\left(\epsilon^{N+1}\right),
\end{aligned}
$$

where

$$
\psi^{[k]}=\left.\frac{1}{k !} \frac{\partial^{k} \psi}{\partial b_{1}^{k}}\right|_{b_{1}=\lambda_{1}}, \quad \phi^{[j]}=\left.\frac{1}{j !} \frac{\partial^{j} \phi}{\partial b_{2}^{j}}\right|_{b_{2}=\lambda_{2}},
$$

with $k, j=0,1,2, \ldots, N$. By a tedious calculation, we find that $\left(\psi^{[k]}, \phi^{[j]}\right)^{\top}$ are analytical and infinitely many eigenfunctions of the Lax pair equation (2) are associated with the zero seed solution; this is a different analytical structure of eigenfunctions of the Lax pair for the BRS. Due to the independence of $\psi$ and $\phi$, these eigenfunctions are classified into two categories, i.e.,

$$
\left(\psi^{[k]}, \phi^{[k]}\right)^{\top}, \quad\left(\psi^{[k]}, \phi^{[j]}\right)^{\top} \quad(k \neq j) .
$$

Note that $\psi^{[k]}$ contains $k+1$ parameters $s_{p}$ (with $p=$ $0,1,2, \ldots, k)$ and $\lambda_{1}$, while $\phi^{[j]}$ contains $j+1$ parameters $s_{p}$ (with $p=0,1,2, \ldots, j$ ) and $\lambda_{2}$. Below, for simplicity, we set $s_{0}>0, s_{j}=0(j \geqslant 1)$, and $\lambda_{1}=-\lambda_{2}^{*}=i \lambda(\lambda \in \mathbb{R})$. Furthermore, hereafter, all results are discussed in a moving reference frame, i.e.,

$$
x^{\prime}=x, \quad y^{\prime}=y-4 \lambda t, \quad r^{\prime}=\sqrt{x^{\prime 2}+y^{\prime 2}},
$$




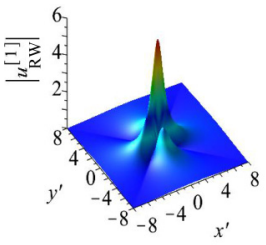

(a)

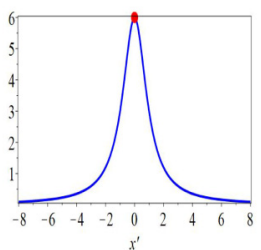

(d)

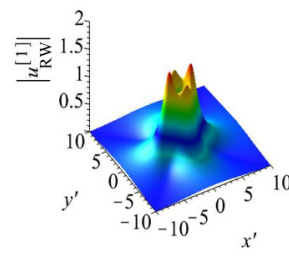

(b)

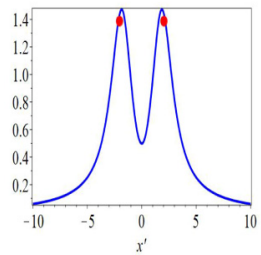

(e)

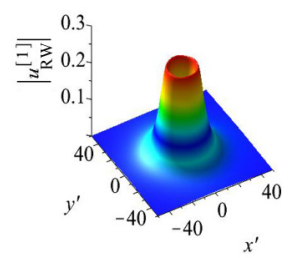

(c)

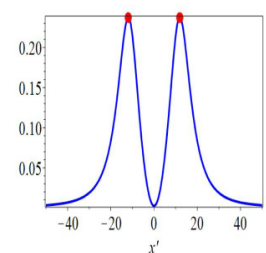

(f)
FIG. 1. (a)-(c) Profiles of the first-order RW $\left|u_{\mathrm{RW}}^{[1]}\right|$ in the $\left(x^{\prime}, y^{\prime}\right)$ plane for $s_{0}=1$ at (a) $t=0$, (b) $t=1$, and (c) $t=200$. (d)-(f) Corresponding cross-sectional profiles of (a)-(c) along the $x^{\prime}$ axis at (d) $t=0$, (e) $t=1$, and (f) $t=200$. The red circles are plotted using the exact result $\frac{6}{s_{0}}$ for $t=0$ and the approximate formulas $r^{\prime}$ and $\left|u_{\mathrm{RW} \text { circle }}^{[1]}\right|$ for $t \neq 0$.

which is more convenient in order to investigate the properties of the obtained solutions.

To derive rational solutions $u^{[N]}$ of the BRS, we select $\Psi_{k}=\left(\psi_{k}, \phi_{k}\right)^{\top}=\left(\psi^{[2 k-1]}, \phi^{[2 k-1]}\right)^{\top}(k=1,2, \ldots, N)$ in Eq. (4). Setting $N=1$ and $\lambda=1$, Eq. (4) yields a usual first-order lump

$$
u_{\text {lump }}^{[1]}=-\frac{2 s_{0} e^{2 i\left(2 t-y^{\prime}\right)}}{x^{\prime 2}+y^{\prime 2}+s_{0}^{2}}
$$

and its maximum value $\left|u_{\text {lump } M}^{[1]}\right|=\frac{2}{s_{0}}$. Setting $N=2$ and $\lambda=1$, Eq. (4) yields a second-order rational solution $u^{[2]}$, corresponding to the first-order RW of the BRS, namely,

$$
u_{\mathrm{RW}}^{[1]}=\frac{6 s_{0} N_{\mathrm{RW}}}{D_{\mathrm{RW}}} e^{-2 i\left(2 t+y^{\prime}\right)},
$$

where

$$
\begin{aligned}
N_{\mathrm{RW}}= & -x^{\prime 4}+y^{\prime 4}-2 s_{0}^{2} x^{\prime 2}+4 s_{0}^{2} y^{\prime 2}-s_{0}^{4} \\
& +12 i t\left(x^{\prime 2}+y^{\prime 2}+s_{0}^{2}\right), \\
D_{\mathrm{RW}}= & \left(x^{\prime 2}+y^{\prime 2}+s_{0}^{2}\right)^{3}+12 s_{0}^{2}\left(x^{\prime 2}+s_{0}^{2}\right) y^{\prime 2}+144 s_{0}^{2} t^{2} .
\end{aligned}
$$

The solution $u_{\mathrm{RW}}^{[1]}$ is a smooth and nonsingular RW as $s_{0}>0$ on zero background, with the following properties.

(a) The modulus $\left|u_{\mathrm{RW}}^{[1]}\right|$, which is an even function of its arguments, decays algebraically like $O\left(\frac{1}{r^{2}}\right)$ for any given time $t$.

(b) When $r^{\prime}=\sqrt{x^{\prime 2}+y^{\prime 2}}=0$, the RW solution $u_{\mathrm{RW}}^{[1]}$ reduces to

$$
u_{\mathrm{RW} c}^{[1]}=\frac{\left(-6 s_{0}^{3}+72 i s_{0} t\right) e^{-4 i t}}{s_{0}^{4}+144 t^{2}} .
$$

Notice that $\left|u_{\mathrm{RW} c}^{[1]}\right|$ reaches the maximum value $\frac{6}{s_{0}}$ at $t=0$ [see Figs. 1(a) and 1(d)], while it bears a (local) minimum value thereafter, and finally decays like $O\left(\frac{s_{0}}{2 t}\right) \sim 0$ as $|t| \rightarrow \infty$. (c) When $r^{\prime} \gg 0$, the maxima of $\left|u_{\mathrm{RW}}^{[1]}\right|$ form a rectangular perimeter profile at an intermediate stage [see, most notably, Fig. 1(b) and also the associated cross section in Fig. 1(e)], which eventually morphs into a circle (i.e., a nearly radial pattern) at the final stage of the evolution [Figs. 1(c) and 1(f)]. At its maximum, the radius of this circle is given by

$$
r^{\prime}=\sqrt{x^{\prime 2}+y^{\prime 2}} \sim\left(6 s_{0} \sqrt{2}|t|\right)^{1 / 3}
$$

as $|t| \rightarrow \infty$, while the corresponding intensity

$$
\left|u_{\mathrm{RW} \text { circle }}^{[1]}\right|^{2} \sim \frac{4}{\left(3 s_{0}|t|\right)^{2 / 3}} \sim \frac{8}{\left(r^{\prime}\right)^{2}} \rightarrow 0
$$

as $|t| \rightarrow \infty$ and $r^{\prime} \rightarrow \infty$.

The symmetry and extreme values of the intensity with $s_{0}=1$ can be verified by the snapshots shown in Fig. 1, for $t=0,1,200$. Note that only profiles for $t \geqslant 0$ are shown because $\left|u_{\mathrm{RW}}^{[1]}\right|$ is an even function of $t$. The red circles denote the maxima of $\left|u_{\mathrm{RW}}^{[1]}\right|$, which are plotted using the exact result $\frac{6}{s_{0}}$ for $t=0$ and the approximate formulas $r^{\prime}$ and $\left|u_{\mathrm{RW} \text { circle }}^{[1]}\right|$ for $t \neq 0$. There exists a deviation between the red circles and the maxima in Fig. 1(e) because time $t$ is too small for the asymptotics to be valid. This deviation disappears in Fig. 1(f) for (sufficiently) large time $t=200$, which is well within the asymptotic regime of Eq. (10).

An animation is provided in the Supplemental Material [40] showing the dynamical evolution of the first-order RW, which can be summarized as follows. At the early stage of the evolution, for large and negative $t$, $\left|u_{\mathrm{RW}}^{[1]}\right|$ appears from the background as a wide circle of low intensity; gradually, it converges to a rectangular column with four maxima at the intermediate stage and then a large peak at $t=0$. Next the RW follows the reverse path, initially dispersing and eventually reverting to a nearly radial form for large and positive $t$.

Here it should be pointed out that the first-order $\mathrm{RW} u_{\mathrm{RW}}^{[1]}$ is very different from the multipole lump [35] of the focusing Davey-Stewartson II equation (or the BRS), because the latter has a nonvanishing amplitude as $|t| \rightarrow \infty$. In addition, this RW solution is naturally also different from the line RW [39] of the focusing DS II (or of the BRS), since the latter is not doubly localized in both $x$ and $y$, but only in one of the two (in the other, it features a line profile).

Extending this approach to $N=3$, Eq. (4) yields a thirdorder rational solution RW-lump solution $u_{\mathrm{RW}}$-lump of the BRS. The explicit expression of $u_{\text {RW-lump }}$ is given in the Appendix. Here we describe its principal dynamical properties.

(i) The modulus $\left|u_{\mathrm{RW}-\text { lump }}\right|$, which is an even function, decays algebraically like $O\left(\frac{1}{r^{\prime 2}}\right)$ for any given time $t$.

(ii) When $r^{\prime}=0$, the solution $u_{\mathrm{RW}-l u m p}$ reduces to

$$
u_{\mathrm{RW}-\mathrm{lump} c}=\frac{12\left(-s_{0}^{4}+60 t^{2}+10 i s_{0}^{2} t\right) e^{-4 i \lambda^{2} t}}{s_{0}\left(s_{0}^{4}+360 t^{2}+60 i s_{0}^{2} t\right)} .
$$

The modulus $\left|u_{\mathrm{RW}-\mathrm{lump} c}\right|$ reaches the maximum value $\frac{12}{s_{0}}$ at $t=$ 0 [Figs. 2(a) and 2(d)], as a result of the interaction of the RW and the lump. Then it returns to maximum value of the first-order lump, i.e., $\left|u_{\text {lumpM }}^{[1]}\right| \sim \frac{2}{s_{0}}$, as the RW disappears for $|t| \rightarrow \infty$. 


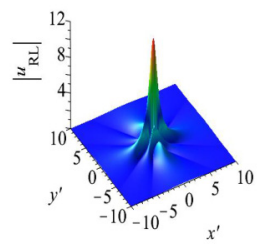

(a)

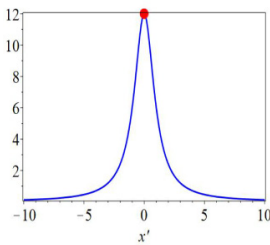

(d)

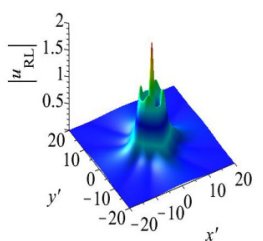

(b)

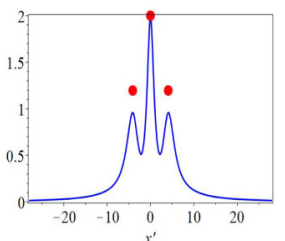

(e)

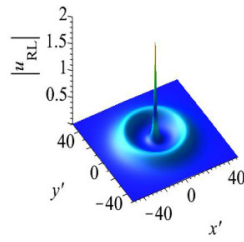

(c)

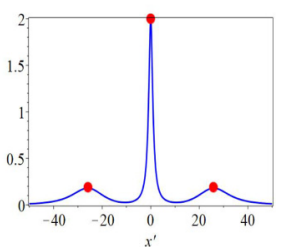

(f)
FIG. 2. (a)-(c) Profiles of the rogue wave-lump $\left|u_{\mathrm{RW}-l u m p}\right|\left(\left|u_{\mathrm{RL}}\right|\right.$ in the figure for convenience) in the $\left(x^{\prime}, y^{\prime}\right)$ plane for $s_{0}=\lambda=1$ at (a) $t=0$, (b) $t=2$, and (c) $t=200$. (d)-(f) Corresponding crosssectional profiles of (a)-(c) along the $x^{\prime}$ axis at (d) $t=0$, (e) $t=2$, and (f) $t=200$. The red circles are plotted using the exact formulation $\frac{12}{s_{0}}$ for $t=0$ and the approximate formulas $\frac{2}{s_{0}}\left(r^{\prime}=0\right)$ and $\frac{2 \sqrt{6}}{r^{\prime}}$ $\left(r^{\prime} \neq 0\right)$ for $t \neq 0$.

(iii) When $r^{\prime} \gg 0$, maxima of $\left|u_{\mathrm{RW}-\text { lump }}\right|$ form a rectangular perimeter profile at the intermediate stage [Figs. 2(b) and 2(e)], which subsequently reverts to a radial outgoing structure at the final stage [Figs. 2(c) and 2(f)] of the evolution, in addition to the persisting lump at the center. At its maximum, the radius of this circle is given by

$$
r^{\prime}=\sqrt{x^{\prime 2}+y^{\prime 2}} \sim\left(288 s_{0}|t|^{2}\right)^{1 / 5}
$$

as $|t| \rightarrow \infty$, while the intensity at this circle is

$$
\left|u_{\text {RW-lump-circle }}\right|^{2} \sim \frac{2 \sqrt{3}^{2 / 5}}{\left(s_{0}|t|^{2}\right)^{2 / 5}} \sim \frac{24}{\left(r^{\prime}\right)^{2}}
$$

as $|t| \rightarrow \infty$ and $r^{\prime} \rightarrow \infty$. It is worth observing that the maxima of $\left|u_{\text {RW-lump }}\right|$ can be decomposed as

$$
\left|u_{\text {lump } M}^{[1]}\right|+\left|u_{\text {RW-lump-circle }}\right| \sim \frac{2}{s_{0}}+\frac{2 \sqrt{6}}{r^{\prime}}
$$

for $|t| \gg 0$, supporting the asymptotic decomposition into a lump and a RW as discussed above. Figure 2 presents the relevant features through the snapshots of different times $(t=0$, 2 , and 200), incorporating, where possible, predictions of the analytical formula, such as the location of the RW circle for large $t$. The animation provided in [40] shows the dynamical evolution of the RW lump. One can discern that (a) a lump in the center always exists; (b) a RW appears as an outer ring from the background at an early stage and then converges gradually to the origin of coordinate as a large peak; (c) later, the RW is dispersed to a circle and finally disappears into the background again as $|t| \rightarrow \infty$.

Higher-order RWs and RW lumps of the BRS can be constructed by evenfold and oddfold DTs, respectively. For instance, a second-order RW $\left|u_{\mathrm{RW}}^{[2]}\right|$, which is constructed by

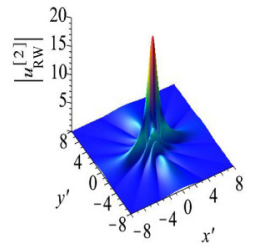

(a)

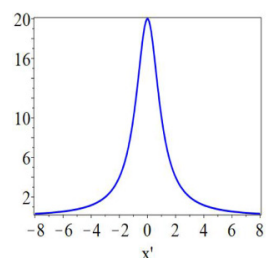

(d)

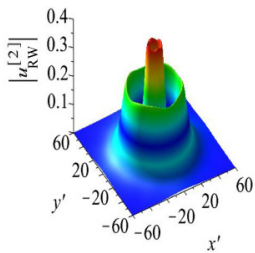

(b)

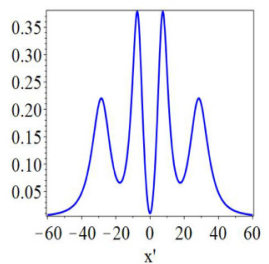

(e)

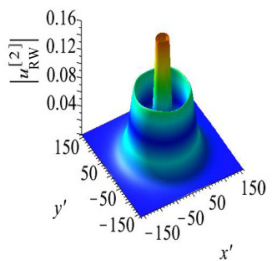

(c)

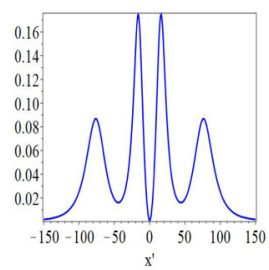

(f)
FIG. 3. (a)-(c) Profiles of the second-order RW $\left|u_{\mathrm{RW}}^{[2]}\right|$ in the $\left(x^{\prime}, y^{\prime}\right)$ plane for $s_{0}=\lambda=1$ at (a) $t=0$, (b) $t=100$, and (c) $t=$ 1000. (d)-(f) Corresponding cross-sectional profiles of (a)-(c) along the $x^{\prime}$ axis at (d) $t=0$, (e) $t=100$, and (f) $t=1000$.

setting $N=4$ in Eq. (4), is plotted in Fig. 3 (see also an animation in [40]) with $s_{0}=\lambda=1$. Both Fig. 3 and the animation demonstrate again the appearance, convergence (to $r^{\prime}=0$ ), dispersion, and disappearance of the RW. Compared with $u_{\mathrm{RW}}^{[1]}$, the second-order RW has (i) higher amplitude and (ii) two rings of intensity maxima for large time $t$. Between them, these harbor a 2D RW hole [see Figs. 3(e) and 3(f)] during the evolution process. It is interesting to note that a 1D RW hole of the NLS equation has been experimentally observed in a water tank [41].

\section{CONCLUSION}

We have reported an analytical structure of eigenfunctions of the Lax pair associated with the zero seed solution for a variant of the Benney-Roskes system. Substituting these eigenfunctions into the evenfold Darboux transformation, we found genuine 2D rogue waves of the BRS. We have thus addressed the long-standing problem of the construction of genuine RWs on zero background, decaying algebraically in both space and time. This way, we provided a proper candidate to describe RWs by means of a canonical 2D generalization of the Peregrine soliton solution of the traditional 1D NLS equation.

As noted above, the RWs were found to be localized in both space and time, that is, as $|t|$ and $r^{\prime} \rightarrow \infty$, their maximum amplitude decayed to 0 as $\frac{1}{r^{\prime}}$. As a by-product, RW-lump solutions of the BRS have been shown to be generated by oddfold DT, which is localized in space only, because one lump of this solution located at the origin approaches $\frac{2}{s_{0}}$ as $|t| \rightarrow \infty$. Multiring RWs (obtained through higher-order expansions) also exist and feature RW holes between them.

We expect that these findings may motivate research efforts towards the generation of 2D RWs in optical systems, in a multidimensional extension of recent $1 \mathrm{D}$ experimental 
efforts, and pave new directions for RW research. Moreover, much like line solitons of the Kadomtsev-Petviashvili II equation, which have been used to explain shallow water wave patterns [42], the RWs of the BRS can also be used for similar studies in optical or possibly atomic physics settings. Additionally, one can also expect that systems described by perturbed BRS or DS II models may still feature some of the relevant phenomenology, as is the case, e.g., with the Peregrine soliton solution and perturbations of the NLS model [43-45].

\section{ACKNOWLEDGMENTS}

This work was supported by the National Natural Science Foundation of China (Grants No. 11671219 and No. 11871446) and the Natural Science Foundation of Zhejiang Province (Grants No. LZ19A010001 and No. LSY19A010002). P.G.K. and D.J.F. acknowledge that this work was made possible by NPRP Grant No. 8-764-1-160 from Qatar National Research Fund (a member of Qatar Foundation).

\section{APPENDIX}

1. Two determinants $\delta_{1}$ and $\delta_{2}$

Two determinants $\delta_{1}$ and $\delta_{2}$ are used in the $N$-fold DT, namely,

$$
\delta_{1}=\left|\begin{array}{cccccc}
\partial_{x}^{N-1} \psi_{1} & \cdots & \partial_{x}^{N-1} \psi_{N} & \partial_{x}^{N-1} \phi_{1}^{*} & \cdots & \partial_{x}^{N-1} \phi_{N}^{*} \\
\vdots & \vdots & \vdots & \vdots & \vdots & \vdots \\
\psi_{1} & \cdots & \psi_{N} & \phi_{1}^{*} & \cdots & \phi_{N}^{*} \\
\partial_{x}^{N-1} \phi_{1} & \cdots & \partial_{x}^{N-1} \phi_{N} & -\partial_{x}^{N-1} \psi_{1}^{*} & \cdots & -\partial_{x}^{N-1} \psi_{N}^{*} \\
\partial_{x}^{N-2} \phi_{1} & \cdots & \partial_{x}^{N-2} \phi_{N} & -\partial_{x}^{N-2} \psi_{1}^{*} & \cdots & -\partial_{x}^{N-2} \psi_{N}^{*} \\
\vdots & \vdots & \vdots & \vdots & \vdots & \vdots \\
\phi_{1} & \cdots & \phi_{N} & -\psi_{1}^{*} & \cdots & -\psi_{N}^{*},
\end{array}\right|
$$

and $\delta_{2}$ is the $N+1$ row of $\delta_{1}$ replaced by a row vector $\eta=\left(\partial_{x}^{N} \psi_{1}, \ldots, \partial_{x}^{N} \psi_{N}, \partial_{x}^{N} \phi_{1}^{*}, \ldots, \partial_{x}^{N} \phi_{N}^{*}\right)$, which are given by Eq. (49) in Ref. [38].

\section{Rogue wave-lump solution of the Benney-Roskes system}

The rogue wave-lump solution $u_{\mathrm{RW}}$-lump of the Benney-Roskes system is generated by a threefold DT, which is expressed explicitly as

$$
u_{\mathrm{RW}-\text { lump }}=\frac{12 s_{0} N_{\mathrm{RW}-\text { lump }}}{D_{\mathrm{RW}-\text { lump }}} e^{-2 i \lambda\left(2 \lambda t+y^{\prime}\right)} \text {, }
$$

where

$$
\begin{aligned}
\operatorname{Re}\left(N_{\mathrm{RW}-l u m p}\right)= & -s_{0}^{10}+\left(20 y^{\prime 2}-5 x^{\prime 2}\right) s_{0}^{8}+\left(65 y^{\prime 4}+60 x^{\prime 2} y^{\prime 2}-10 x^{\prime 4}+300 t^{2}\right) s_{0}^{6} \\
& -\left[10 x^{\prime 6}-60 y^{\prime 2} x^{\prime 4}-\left(135 y^{\prime 4}+900 t^{2}\right) x^{\prime 2}+75 y^{\prime 6}-4500 y^{\prime 2} t^{2}\right] s_{0}^{4} \\
& -\left[5 x^{\prime 8}-20 x^{\prime 6} y^{\prime 2}-\left(75 y^{\prime 4}+900 t^{2}\right) x^{\prime 4}-\left(10 y^{\prime 6}+5400 y^{\prime 2} t^{2}\right) x^{\prime 2}+10 y^{\prime 8}-900 t^{2} y^{\prime 4}-21600 t^{4}\right] s_{0}^{2} \\
& -\left(x^{\prime 4}-3 x^{\prime 2} y^{\prime 2}+y^{\prime 4}-300 t^{2}\right)\left(x^{\prime 2}+y^{\prime 2}\right)^{3} \\
\operatorname{Im}\left(N_{\mathrm{RW}-\operatorname{lump}}\right)= & 10 t\left(7 s_{0}^{8}+24 s_{0}^{6} x^{\prime 2}+66 s_{0}^{6} y^{\prime 2}+30 s_{0}^{4} x^{\prime 4}+90 s_{0}^{4} x^{\prime 2} y^{\prime 2}-60 y^{\prime 4} s_{0}^{4}+16 s_{0}^{2} x^{\prime 6}+30 s_{0}^{2} x^{\prime 4} y^{\prime 2}\right. \\
& \left.-60 s_{0}^{2} y^{\prime 4} x^{\prime 2}-26 s_{0}^{2} y^{\prime 6}+3 x^{\prime 8}+6 x^{\prime 6} y^{\prime 2}-6 y^{\prime 6} x^{\prime 2}-3 y^{\prime 8}\right) \\
D_{\mathrm{RW}-l u m p} & \left(x^{\prime 2}+y^{\prime 2}+s_{0}^{2}\right)^{6}+6 s_{0}^{2}\left\{2160\left(s_{0}^{2}+x^{\prime 2}+y^{\prime 2}\right) t^{4}+24\left[\frac{7}{5} s_{0}^{6}+10 s_{0}^{2}\left(y^{\prime 2}-\frac{2}{5} s_{0}^{2}\right)^{2}+7 s_{0}^{4} x^{\prime 2}+5 s_{0}^{2} x^{\prime 4}+x^{\prime 6}+y^{\prime 6}\right] t^{2}\right. \\
& +s_{0}^{8} y^{\prime 2}+4 s_{0}^{6} x^{\prime 2} y^{\prime 2}+2 s_{0}^{6} y^{\prime 4}+6 s_{0}^{4} x^{\prime 4} y^{\prime 2}+6 s_{0}^{4} x^{\prime 2} y^{\prime 4}+10 s_{0}^{4} y^{\prime 6}+4 s_{0}^{2} x^{\prime 6} y^{\prime 2} \\
& \left.+6 s_{0}^{2} x^{\prime 4} y^{\prime 4}+12 s_{0}^{2} x^{\prime 2} y^{\prime 6}+s_{0}^{2} y^{\prime 8}+x^{\prime 8} y^{\prime 2}+2 x^{\prime 6} y^{\prime 4}+2 x^{\prime 4} y^{\prime 6}+x^{\prime 2} y^{\prime 8}\right\}
\end{aligned}
$$

with $\mathrm{Re}$ and Im denoting the real and imaginary parts of $N_{\mathrm{RW}-l u m p}$, respectively. It is clear that this rational solution is smooth and nonsingular as $s_{0} \neq 0$. Note that $s_{0}$ and $\lambda$ are two real constants. 
[1] M. Onorato, S. Residori, U. Bortolozzo, A. Montinad, and F. T. Arecchi, Phys. Rep. 528, 47 (2013).

[2] S. Chen, F. Baronio, J. M. Soto-Crespo, P. Grelu, and D. Mihalache, J. Phys. A: Math. Theor. 50, 463001 (2017).

[3] E. Pelinovsky and C. Kharif, Extreme Ocean Waves (Springer, Berlin, 2008).

[4] K. Dysthe, H. E. Krogstad, and P. Müller, Annu. Rev. Fluid Mech. 40, 287 (2008).

[5] A. Osborne, Nonlinear Ocean Waves and the Inverse Scattering Transform (Elsevier, New York, 2010).

[6] Rogue and Shock Waves in Nonlinear Dispersive Media, edited by M. Onorato, S. Resitori, and F. Baronio (Springer, Berlin, 2016).

[7] D. J. Benney and G. J. Roskes, Stud. Appl. Math. 48, 377 (1969).

[8] A. Davey and K. Stewartson, Proc. R. Soc. London Ser. A 338, 101 (1974)

[9] V. D. Djordjević and L. G. Redekopp, J. Fluid Mech. 79, 703 (1977).

[10] M. J. Ablowitz and H. Segur, J. Fluid Mech. 92, 691 (1979).

[11] M. J. Ablowitz, G. Biondini, and S. Blair, Phys. Rev. E 63, 046605 (2001).

[12] M. Ablowitz, I. Bakirtas, and B. Ilan, Eur. Phys. J.: Spec. Top. 147, 343 (2007).

[13] M. J. Ablowitz, Nonlinear Dispersive Waves: Asymptotic Analysis and Solitons (Cambridge University Press, Cambridge, 2011), Chap. 6.8.

[14] C. Sulem and P. L. Sulem, The Nonlinear Schrödinger Equation (Springer, New York, 1999).

[15] M. J. Ablowitz, B. Prinari, and A. D. Trubatch, Discrete and Continuous Nonlinear Schrödinger Systems (Cambridge University Press, Cambridge, 2004).

[16] D. H. Peregrine, J. Aust. Math. Soc. Ser. B 25, 16 (1983).

[17] N. N. Akhmediev, V. M. Eleonskii, and N. E. Kulagin, Sov. Phys. JETP 62, 894 (1985).

[18] N. Akhmediev, A. Ankiewicz, and M. Taki, Phys. Lett. A 373, 675 (2009).

[19] D. J. Kedziora, A. Ankiewicz, and N. Akhmediev, Phys. Rev. E 84, 056611 (2011).

[20] D. J. Kedziora, A. Ankiewicz, and N. Akhmediev, Phys. Rev. E 88, 013207 (2013).

[21] J. S. He, H. R. Zhang, L. H. Wang, K. Porsezian, and A. S. Fokas, Phys. Rev. E 87, 052914 (2013).

[22] L. H. Wang, J. S. He, H. Xu, J. Wang, and K. Porsezian, Phys. Rev. E 95, 042217 (2017).

[23] B. Kibler, J. Fatome, C. Finot, G. Millot, F. Dias, G. Genty, N. Akhmediev, and J. M. Dudley, Nat. Phys. 6, 790 (2010).

[24] K. Hammani, B. Kibler, C. Finot, P. Morin, J. Fatome, J. M. Dudley, and G. Millot, Opt. Lett. 36, 112 (2011).

[25] J. M. Dudley, F. Dias, M. Erkintalo, and G. Genty, Nat. Photon. 8, 755 (2014).

[26] B. Frisquet, B. Kibler, P. Morin, F. Baronio, M. Conforti, and B. Wetzel, Sci. Rep. 6, 20785 (2016).

[27] A. Chabchoub, N. P. Hoffmann, and N. Akhmediev, Phys. Rev. Lett. 106, 204502 (2011).
[28] A. Chabchoub, N. Hoffmann, M. Onorato, A. Slunyaev, A. Sergeeva, E. Pelinovsky, and N. Akhmediev, Phys. Rev. E 86, 056601 (2012).

[29] A. Chabchoub, Phys. Rev. Lett. 117, 144103 (2016).

[30] A. R. Osborne, M. Onorato, and M. Serio, Phys. Lett. A 275, $386(2000)$.

[31] A. Kundu, A. Mukherjee, and T. Naskar, Proc. R. Soc. A 470, 20130576 (2014).

[32] A. S. Fokas and M. J. Ablowitz, J. Math. Phys. 25, 2494 (1984).

[33] Important Developments in Soliton Theory, edited by A. S. Fokas and V. E. Zakharov (Springer, Berlin, 1993).

[34] M. J. Ablowitz and P. A. Clarkson, Solitons, Nonlinear Evolution Equations and Inverse Scattering (Cambridge University Press, Cambridge, 1991).

[35] J. Villarroel and M. J. Ablowitz, SIAM J. Math. Anal. 34, 1253 (2003).

[36] V. B. Matveev and M. A. Salle, Darboux Transformations and Solitons (Springer, Berlin, 1991).

[37] C. H. Gu, H. S. Hu, and Z. X. Zhou, Darboux Transformations in Integrable Systems: Theory and Their Applications to Geometry (Springer, Berlin, 2005).

[38] X. Kou, Rogue wave solutions to integrable system by Darboux transformation, M.Sc. thesis, University of Vermont, 2014.

[39] Y. Ohta and J. Yang, J. Phys. A: Math. Theor. 46, 105202 (2013).

[40] See Supplemental Material at http://link.aps.org/supplemental/ 10.1103/PhysRevResearch.2.033376 for dynamical evolution. More specifically, we give there (1) the movie 13-3Danimationrw.gif, which shows the dynamical evolution of the first-order RW given by Eq. (8) with the parameter $s_{0}=1$ from $t=-200$ to $t=200$, and the movie 13-3Danimationrw1.gif, which clearly illustrates the dynamical evolution process of the intermediate stage $(t \sim 0)$; $(2)$ the movie 135-3Danimationrwlump.gif, which displays the dynamical evolution of the rogue wave-lump

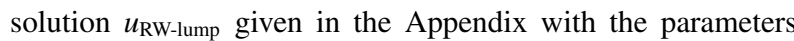
$\lambda=1$ and $s_{0}=1$ from $t=-200$ to $t=200$, and the movie 135-3Danimationrwlump1.gif, which clearly illustrates the dynamical evolution process of the intermediate stage $(t \sim 0)$; and (3) the movie 1357-3Danimation2rw.gif, which shows the dynamical evolution of the second-order RW of the BRS with the parameters $\lambda=1$ and $s_{0}=1$ from $t=-1000$ to $t=1000$, and the movie 1357-3Danimation2rw1.gif, which clearly illustrates the dynamical evolution process of the intermediate stage $(t \sim 0)$.

[41] A. Chabchoub, N. P. Hoffmann, and N. Akhmediev, J. Geophys. Res. 117, C00J02 (2012).

[42] M. J. Ablowitz and D. E. Baldwin, Phys. Rev. E 86, 036305 (2012).

[43] A. Armaroli, M. Brunetti, and J. Kasparian, Phys. Rev. E 96, 012222 (2017).

[44] M. Taki, A. Mussot, A. Kudlinski, E. Louvergneaux, M. Kolobov, and M. Douay, Phys. Lett. A 374, 691 (2010).

[45] A. Armaroli, C. Conti, and F. Biancalana, Optica 2, 497 (2015). 